\title{
Questes
}

Revue pluridisciplinaire d'études médiévales

Journée d'étude 1 - Trier, classer, organiser | 2022

Trier, classer, organiser

\section{Entre herbe et arbre. Classer les végétaux au statut ambivalent dans la littérature encyclopédique médiévale}

Alice Laforêt

\section{OpenEdition}

Journals

Édition électronique

URL : https://journals.openedition.org/questes/5980

DOI : 10.4000 /questes.5980

ISSN : 2109-9472

Éditeur

Les Amis de Questes

Édition imprimée

Pagination : 105-118

ISSN : 2102-7188

Référence électronique

Alice Laforêt, « Entre herbe et arbre. Classer les végétaux au statut ambivalent dans la littérature

encyclopédique médiévale », Questes [En ligne], Journée d'étude 1 - Trier, classer, organiser | 2022, mis en ligne le 31 janvier 2022, consulté le 21 février 2022. URL : http://journals.openedition.org/questes/ 5980 ; DOI : https://doi.org/10.4000/questes.5980 


\title{
Entre herbe et arbre. \\ Classer les végétaux au statut ambivalent dans la littérature encyclopédique médiévale
}

\author{
Alice LAFORÊT
}

«Moyen entre herbe et arbre $^{1}$ »: c'est ainsi qu'est qualifié le roseau dans le Livre des propriétés des choses, traduction française réalisée par Jean Corbechon en 1372 du De proprietatibus rerum de Barthélemy l'Anglais. Définie de telle manière, cette plante échappe à une classification rigoureuse. Or il n'est pas rare de rencontrer, dans le savoir botanique médiéval ${ }^{2}$, des végétaux à l'identité ambivalente. Ni herbe, ni arbre, ni arbrisseau, leur définition est révélatrice des pratiques de travail et de la conception du monde végétal des naturalistes de la période-et parmi eux, de ceux que l'historiographie qualifie d'encyclopédistes ${ }^{3}$. Ces derniers ont pour projet, dans les compilations

\footnotetext{
${ }^{1}$ Paris, BnF, fr. 16993, f. 245. Dans le texte latin : «Arundo est media inter herbam et arborem », Barthélemy l'Anglais, De proprietatibus rerum, XVII, c. 7, p. 37.

${ }^{2}$ Comme le souligne Bernard Ribémont, «parler de botanique au Moyen Âge est toujours quelque peu périlleux » (Le Moyen Âge et la science : approche de quelques disciplines et personnalités scientifiques médiévales, Paris, Klincksieck, 1991,

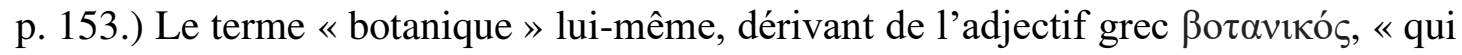
se rapporte aux plantes ", ne s'impose pas comme substantif avant le XVII ${ }^{\mathrm{e}}$ siècle. Son usage appliqué aux sciences médiévales est donc anachronique, nous l'emploierons notamment par commodité pour désigner les textes - encyclopédies et herbiers - qui traitent des plantes et de leurs propriétés.

${ }^{3}$ Le terme «naturaliste » est ici employé pour désigner les auteurs d'un corpus de textes consacrés à la description méthodique des réalités naturelles. Comme le rappelle Isabelle Draelants, les auteurs des encyclopédies latines du XIII siècle « ont en commun l'étude de la nature et, en grande partie, un même type de documentation. Leurs auteurs [...] sont des "naturalistes" »-l'équivalent de Naturphilosoph ou de
} 
qu'ils réalisent, d'organiser le monde naturel selon des catégories rigoureusement circonscrites. Ordonner, tel est le travail de l'encyclopédiste: Vincent de Beauvais va jusqu'à affirmer dans le prologue de son Speculum maius que peu d'éléments proviennent de ses connaissances propres et que l'ordonnancement des connaissances est son seul apport : « rares, voire nuls sont les éléments qui proviennent de moi. C'est à eux [aux auteurs cités par l'encyclopédiste] que revient l'autorité et à moi seulement l'organisation ${ }^{4} \gg$.

Afin d'aborder cette volonté d'ordonnancement de la flore, nous nous pencherons sur la classification des végétaux ambivalents, au sein d'un corpus de textes latins cohérent, mais non exhaustif. Les trois principales compilations encyclopédiques du $\mathrm{XIII}^{\mathrm{e}}$ siècle que sont le $D e$ proprietatibus rerum de Barthélemy l'Anglais (rédigé vers 1240), le Liber de natura rerum de Thomas de Cantimpré (achevé vers 1237-1240) ainsi que le Speculum naturale (écrit par Vincent de Beauvais vers 12571258) serviront de base à l'étude ${ }^{5}$ : dans chacune d'entre elles se trouvent des livres spécifiquement dédiés au monde végétal. Des comparaisons seront ponctuellement faites avec des textes qui n'appartiennent pas à proprement parler au genre encyclopédique, mais dont la manière d'aborder les plantes permet de les rapprocher des ouvrages

\footnotetext{
physiologos n'existant pas en français » (Isabelle Draelants « La science naturelle et ses sources chez Barthélemy l'Anglais et les encyclopédistes contemporains », dans Bartholomaeus Anglicus, "De proprietatibus rerum». Texte latin et réception vernaculaire, Turnhout, Brepols, 2005, p. 43-99, voir p. 43).

${ }^{4}$ « ex meo pauca vel quasi nulla. Ipsorum igitur est auctoritate, nostrum autem sola parcium ordinatione »Vincent de Beauvais, Libellus apologeticus, 3 (Dijon, BM, 568) Les différentes versions du prologue du Speculum Maius sont consultables en ligne : http://atilf.atilf.fr/bichard/scripts/artem2/initvdb.exe (consulté le 26 mai 2017). ${ }^{5}$ Toutes les citations sont issues des éditions suivantes : Barthélemy l'Anglais, De proprietatibus rerum, Volume VI: Liber XVII, éd. Iolanda Ventura, Turnhout, Brepols, 2007 ; Thomas de Cantimpré, Liber de natura rerum: editio princeps secundum codices manuscriptos, éd. Helmut Boese, Berlin, De Gruyter, 1973 ; Vincent de Beauvais, Bibliotheca mundi, Speculum quadruplex, Naturale, Doctrinale, Morale, Historiale, éd. John Jones, Duaci, B. Belleri, 1624.
} 
précédemment cités. Il pourra s'agir de textes de matière médicale, tels que le Tractatus de herbis (dont le plus ancien témoin conservé date de la fin du XIII ${ }^{\mathrm{e}}$ siècle ${ }^{6}$ ), de la grande somme sur les plantes réalisée par Albert le Grand vers le milieu du XIII ${ }^{\mathrm{e}}$ siècle ${ }^{7}$, ou encore du traité agronomique $\mathrm{du}$ début du $\mathrm{XIV}^{\mathrm{e}}$ siècle qu'est le Ruralia commoda de Pierre de Crescens ${ }^{8}$. Ces textes ont en commun de relever du schéma de l'herbier, défini comme un ouvrage traitant des plantes et de leurs propriétés, de manière ordonnée et dans une perspective de totalisation du savoir, suivant l'héritage de l'œuvre du médecin grec Dioscoride. L'ordonnancement de la flore passe par la définition de grandes catégories du monde végétal, qui se heurte néanmoins à des essences aux identités ambiguës. À travers l'étude de plantes particulièrement difficiles à classer, nous tenterons de cerner les enjeux de la mise en ordre des connaissances par les naturalistes médiévaux. Ces derniers se confrontent en effet à une nature foisonnante. Dans le Liber de natura rerum de Thomas de Cantimpré, trois livres (X à XII) sont consacrés à la flore : cela représente un total de cent dix-sept chapitres. Le livre XVII du De proprietatibus rerum de Barthélemy l'Anglais, dédié au monde végétal, se compose de cent quatre-vingt-quatorze chapitres. Dans la somme monumentale qu'est le Speculum naturale, les livres IX à XIV concernent les plantes, ce qui représente un total de huit cent vingt-huit chapitres. Il relève donc du projet encyclopédique de rationaliser la présentation de ces connaissances et d'ordonner ces listes de plantes que constituent les chapitres dédiés au monde végétal.

\footnotetext{
${ }^{6}$ Ps. Bartholomaeus Mini de Senis, Tractatus de herbis (MS London, British Library, Egerton 747), éd. Iolanda Ventura, Firenze, SISMEL-Ed. del Galluzzo, 2009.

${ }^{7}$ Albert le Grand, De vegetabilibus libri VII, éd. Ernst Meyer et Carl Jessen, Berlin, Georg Reimer, 1867.

8 Pierre de Crescens, Ruralia Commoda, éd. Will Richter et Reinhilt RichterBergmeier, Heidelberg, C. Winter, 1995-2002, 4 vol.
} 


\section{Nommer, c'est classer : les différentes catégories du monde végétal}

Le savoir botanique médiéval s'articule autour de deux grandes catégories canoniques: les herbes et les arbres. Ces deux types de végétaux structurent souvent les ouvrages sur les plantes. Nous trouvons déjà cette division chez Pline l'Ancien, autorité incontournable pour le savoir botanique médiéval : les livres XII à XVII de l'Historia naturalis traitent des arbres, tandis que les livres XVIII à XXII se concentrent plutôt sur les plantes herbacées (céréales, lin, fleurs, herbes et grains). De même, Isidore de Séville, dans ses Étymologies, consacre d'abord le livre XVII aux arbres, puis aux herbes, et place la question spécifique des légumes dans deux chapitres à part ${ }^{9}$. À la suite de ces auctoritates, les encyclopédistes médiévaux usent de ces grandes catégories pour agencer leurs ouvrages. Thomas de Cantimpré évoque tout d'abord les arbres communs, puis les arbres aromatiques (livres X et XI), avant de s'arrêter dans un troisième livre sur les herbes (livre XII). Vincent de Beauvais, quant à lui, divise son propos en quatre livres, consacrés respectivement aux herbes aromatiques (livre IX), aux herbes communes (livre X), aux arbres communs (livre XII), et enfin aux arbres fruitiers et aromatiques (livre XIII). Albert le Grand et Pierre de Crescens consacrent tous deux un chapitre aux arbres puis un chapitre aux herbes ${ }^{10}$. Dans d'autres textes, en revanche, c'est l'ordre alphabétique qui préside : c'est le cas dans les herbiers de matière médicale, comme le Tractatus de herbis ou son adaptation en langue vernaculaire, le Livre des simples médecines. De

\footnotetext{
${ }^{9}$ Il s'agit du chapitre X «De oleribus » ainsi que du chapitre XI, « De odoratis oleribus », consacré aux légumes aromatiques. Isidore de Séville, Étymologies, Livre XVII, De l'agriculture, éd. et trad. Jacques André, Paris, Les Belles Lettres, 1981.

${ }^{10}$ Il s'agit des traités I et II du livre VI dans le De vegetabilibus d'Albert le Grand et des livres V et VI dans l'œuvre de Pierre de Crescens.
} 
même, le livre XVII du De proprietatibus rerum constitue un herbier alphabétique. Dans les textes encyclopédiques comme dans les herbiers de matière médicale, chaque essence est le plus souvent caractérisée, au début du chapitre, par un élément de nomenclature qui rattache la plante à une famille du monde végétal. Dans ce savoir au sein duquel la valeur lexicale est prééminente, les choix de vocabulaire sont bien révélateurs de la réflexion menée sur le monde des plantes. Au-delà de la division binaire entre arbres et herbes que reflète l'agencement des encyclopédies, les compilateurs mettent en œuvre des catégories plus subtiles pour ordonner le monde végétal. Loin de faire preuve d'une absence de classification des végétaux, le Moyen Âge connaît au contraire plusieurs degrés d'organisation, qui demeurent globalement les mêmes dans le savoir botanique moderne. Herba, frutex, arbustum, arbor traduits le plus souvent dans les versions en langue vernaculaire par «herbe», « arbrisseau », « arbuste » et « arbre », sont autant de termes qui agencent et hiérarchisent la flore des encyclopédistes.

La structuration des textes savants en différentes sections ainsi que la nomenclature employée permettent d'ordonner les centaines d'essences présentes dans la littérature botanique. S'interroger sur les différentes catégories du monde végétal suppose bien sûr de se pencher sur des critères morphologiques - la taille $\mathrm{du}$ tronc, l'importance de la ramification, la présence d'écorce sont autant de caractéristiques relevées par les compilateurs. Ces critères, sur lesquels nous ne nous attarderons pas ici, se révèlent néanmoins fragiles et mouvants quand il s'agit de les convoquer pour classer une plante dans telle ou telle catégorie. Pour de nombreuses essences végétales, la description morphologique est d'ailleurs très concise ou inexistante. Dans le savoir naturel médiéval, nommer une essence est l'étape fondatrice de toute classification. Dans une démarche toute tautologique, il est donc possible d'affirmer qu'un 
végétal appartient au règne des arbres dès lors qu'il est traité dans une section consacrée aux arbres ou à laquelle on associe le substantif arboret «arbre» dans les textes en vernaculaire. Ce processus est cependant loin d'être linéaire, et la nomenclature botanique peut s'avérer à géométrie variable.

\section{Classer, c'est choisir : les incertitudes de la nomenclature botanique}

$\mathrm{Si}$ ces catégories du monde végétal héritées du savoir antique peuvent paraître bien établies et d'une grande stabilité, nombreuses sont les plantes, d'un texte à l'autre, qui se soustraient au périmètre de ces typologies. Il n'est pas rare de trouver dans les textes des arbrisseaux «semblables à des arbres", voire des herbes «poussant comme des arbres $»^{11}$, ce qui invalide, de fait, le critère morphologique de la taille quant à la définition de l'arbre, une catégorie qui pourrait pourtant sembler particulièrement uniforme et univoque. L'ambition de livrer une synthèse ordonnée du savoir sur les plantes se heurte à l'hétérogénéité du monde végétal. Dès lors, qu'est-ce qu'un arbre, une herbe ou un arbrisseau au sens médiéval? Quels choix sont effectués par les encyclopédistes pour décrire ces végétaux ? Quelles catégories sont convoquées afin de les classer ? Et quelle appréhension globale du monde végétal ces listes de plantes nous signalent-elles?

Une même essence peut fréquemment passer selon les sources du statut d'arbor à celui de frutex, voire d'herba. C'est le cas du roseau, mais également du myrte, de l'arbre à baume, du sureau ou encore de la vigne - végétal ambivalent s'il en est. La nomenclature botanique peut également faire l'objet d'hésitations au sein d'un même texte. Albert le

\footnotetext{
11 «Agnus castus frutex est similis arbor», Tractatus de herbis, 6, éd. cit., p. 212. «Herba paralisis [...] crescens quasi arbor», ibid., 230, p. 486.
} 
Grand souligne lui-même, alors qu'il commente les catégories de plantes établies par le traité de Nicolas de Damas dont il s'inspire, l'ambivalence de certains végétaux qu'il est difficile d'associer à une catégorie précise, mais qui se situent entre l'arbrisseau et l'arbre. La terminologie botanique est loin d'être constante: un relevé systématique de la nomenclature utilisée pour définir les arbres, dans le corpus textuel que nous avons défini, nous a permis de constater que les trois-quarts des cent vingt plantes traitées étaient qualifiées par le même terme dans chacun des textes ${ }^{12}$, ce qui signifie qu'un quart de ces végétaux présente une terminologie variable d'un texte à l'autre. Souvent, cette incertitude de la nomenclature provient d'une lecture critique des autorités. Le compilateur peut se trouver face à des terminologies variables selon les sources, comme en témoigne un passage du Tractatus de herbis consacré à l'arbre à baume :

L'arbre à baume est un arbre, d'après ce que disent certains, ou un arbrisseau, ce qui est plus proche de la vérité, comme en témoigne Dioscoride, ainsi que d'autres auteurs, qui l'ont $\mathrm{vu}^{13}$.

L'auteur privilégie ici le choix terminologique de Dioscoride, et décide de classer l'arbre à baume parmi les arbrisseaux. Ce même végétal est défini comme un arbre dans d'autres textes - chez Albert le Grand par exemple («L'arbre à baume, comme dit Avicenne, est un arbre qui croît

\footnotetext{
12 Nous avons procédé, dans le cadre d'une thèse d'École des chartes, à une comparaison systématique de la nomenclature botanique employée dans les textes d'un corpus d'ouvrages savants, sous la forme d'un tableau (voir Alice Laforêt, L'Arbre et le livre au Moyen Âge. Encyclopédies et herbiers: les arbres dans le savoir botanique occidental (XIII ${ }^{e} X V^{e}$ siècle), thèse pour le diplôme d'archivistepaléographe, École nationale des chartes, 2016, p. 248-260). Le recensement que nous citons est issu d'une version remaniée de ce tableau. Cette étude ne portait que sur les essences végétales qualifiées d'arbres dans au moins un des textes étudiés. Une étude plus systématique portant sur toutes les catégories de la flore serait donc susceptible de révéler une diversité lexicale encore plus importante.

13 «Balsamus arbor est, ut quidam dicunt, vel frutex, quod verius est, adtestante Diascoride et etiam illis, qui viderunt » Tractatus de herbis, 59, op. cit., p. 274.
} 
en Égypte $\left.{ }^{14} \gg\right)$ ou chez Vincent de Beauvais, qui se réfère pourtant explicitement à Dioscoride dans son texte (« Dioscoride. L'arbre à baume est un arbre assez chaud et sec, qui ressemble au grenadier, doté de branches nombreuses, minces et blanchâtres $\left.{ }^{15} »\right)$. Classer, c'est donc faire un choix parmi les sources disponibles, mais c'est également privilégier une catégorie parmi plusieurs possibles.

La méthodologie adoptée par Vincent de Beauvais est à ce titre particulièrement intéressante. Le compilateur souligne ses apports dans le Speculum maius en les faisant procéder de la mention auctor $^{16}$. Un certain nombre de ses interventions concerne la nomenclature botanique. Vincent de Beauvais mène ainsi une réflexion sur la nature de l'arbre à travers certains cas d'étude. Il déclare par exemple au chapitre sur le romarin :

Dioscoride. Le romarin, que certains appellent lithos, est un arbre aux vertus astringentes, de petite taille et de complexion sèche (...) Auteur. Le romarin est traité plus haut au chapitre sur les herbes sauvages, puisque, tel un arbrisseau, il apparaît comme un intermédiaire entre les arbres et les herbes ${ }^{17}$.

Après avoir exposé l'avis de Dioscoride qui fait du romarin un arbre, le compilateur s'exprime et assimile cette plante à un arbrisseau. Il

14 «Balsamus ut dicit Avicenna, est arbor egyptia» Albert le Grand, De vegetabilibus, 1 . VI, tract. I, 8, p. 354.

15 «Dioscorides. Balsamus est arbor nimis calida et sicca, malogranato similis, ramosa, ramis tenuibus et exalbidis » Vincent de Beauvais, Speculum naturale, 1. XIII, 64.

${ }^{16}$ Ce type de marqueur «auctor », qui souligne que l'élément qui va suivre n'émane pas d'une autorité, mais de l'auteur lui-même, n'a pas son équivalent chez les autres encyclopédistes. Il convient cependant de modérer sa portée, comme le souligne Isabelle Draelants, «sous le vocable d'auctor, peuvent se cacher - ou se déclarer, de la bouche de Vincent de Beauvais - de nombreuses ouvres et auteurs dont il adopte l'opinion ». (Isabelle Draelants « La science naturelle et ses sources chez Barthélemy l'Anglais et les encyclopédistes contemporains », art. cit., p. 63)

17 «Dioscorides. Rosmarinum quod alii lithos vocant: est arbor quaedam stiptica, et mediocriter extendatoria et desiccativa. [...] Auctor. De rosmarino etiam dictum est superius inter herbas incultas, qui tanquam frutex medius esse videtur inter arbores et herbas » Vincent de Beauvais, Speculum naturale, 1. XII, 94. 
précise en outre qu'un propos sur le romarin est également développé au sein du livre consacré aux herbes sauvages ${ }^{18}$, puisqu'il s'agit d'un végétal à mi-chemin entre l'herbe et l'arbre. Vincent de Beauvais pose donc un regard critique sur ses sources et fait preuve d'une précision extrême dans l'ordonnancement de son ouvrage, puisqu'il traite ce végétal ambivalent dans deux livres différents et explicite sa démarche. Le cas du roseau est similaire. Vincent de Beauvais écrit en effet à son sujet :

Auteur. Isidore classe le roseau parmi les arbres, et cela, semble-t-il, du fait de certaines de ses propriétés, car on dit que le roseau est d'une telle taille et épaisseur qu'on peut en fabriquer des navires et des bâtiments. Les autres propriétés du roseau sont traitées plus haut, au chapitre des herbes sauvages et des herbes comestibles ${ }^{19}$.

L'encyclopédiste justifie la démarche d'Isidore de Séville, qui place le roseau parmi les arbres, en raison de certaines de ses propriétés en premier lieu sa taille et l'épaisseur de sa matière, utilisable en construction. C'est dans ce type de passages que se dessine l'arbre archétypal des encyclopédistes. Néanmoins, le roseau demeure à la lisière du monde des arbres, et Vincent de Beauvais ajoute qu'il possède des caractéristiques qui le rapprochent plus de l'herba, et dont l'auteur traite dans le chapitre «De arundine», au sein du livre IX sur les herbes. Les cas du roseau et du romarin reflètent bien l'attention accordée aux questions de nomenclature. C'est aussi le cas du nard, pour lequel le

\footnotetext{
${ }^{18}$ Le chapitre 127 du livre IX, sur les herbes, est en effet consacré au romarin. Ce chapitre s'ouvre sur une citation d'Isidore de Séville, qui décrit bien le romarin comme appartenant à la catégorie des herbes : "Isidorus. Rosmarinum Latini ab effectu herbam salutarem vocant, folia habet feniculi similia atque aspera et rotatim terre prostrata. » Vincent de Beauvais, Speculum naturale, l. IX, 127.

${ }^{19}$ «Auctor. Arundinem ponit Isidorus inter arbores, et hoc (vt videtur) propter quadam speciaes, quarum tanta crassitudo et magnitudo esse dicitur, vt ex illis naues et aedificia fabricentur. De caeteris autem dictum est superius inter herbas incultas et inter sativas » Vincent de Beauvais, Speculum naturale, 1. XII, 67.
} 
compilateur écrit qu'il s'agit d'un frutex, à mi-chemin entre les herbes et les arbres, dont il est aussi question dans le livre des herbes communes. Par ses interventions et ce système de renvois entre les différents livres du Speculum naturale, Vincent de Beauvais fait preuve d'une réflexion très subtile sur les différentes catégories du monde végétal et résout habilement ses hésitations concernant la terminologie.

\section{Agnus castus et herba savina : des plantes ambivalentes}

L'agnus castus (Vitex Agnus Castus L. selon la nomenclature de Linné, gattilier en français moderne) est un arbuste pouvant atteindre des tailles très variables et qui pousse surtout à la faveur du climat méditerranéen. Ses fleurs, bien reconnaissables, forment de longs épis bleu violacé, roses et parfois blancs. Il s'agit d'un exemple de plante commune dans le savoir médiéval, car traité par presque tous les ouvrages de notre corpus, des herbiers de matière médicale aux encyclopédies. Le nom latin de l'agnus castus est un véritable jeu de mots, en lien avec les propriétés anaphrodisiaques qui lui sont prêtées. Ses branches auraient en effet le pouvoir d'éteindre les ardeurs sexuelles. Dans la pharmacopée médiévale, cette plante vaut également contre l'hydropisie et diverses maladies féminines.

Cette plante est un exemple particulièrement caractéristique de l'identité ambivalente de certains végétaux. Un relevé systématique du lexique utilisé pour la qualifier dans les ouvrages de notre corpus permet d'en rendre compte. On note en premier lieu qu'aucune des trois grandes compilations encyclopédiques du XIII ${ }^{\mathrm{e}}$ siècle ne s'accorde à son sujet : Barthélemy 1'Anglais qualifie l'agnus castus d'herba ${ }^{20}$, Vincent

\footnotetext{
${ }^{20}$ Barthélemy l'Anglais, De proprietatibus rerum, 1. XVII, c. 15, p. 46.
} 
de Beauvais de frutex $^{21}$, tandis que Thomas de Cantimpré le classe parmi les arbres ${ }^{22}$. Un examen des autres sources du savoir botanique médiéval confirme cette diversité lexicale. Dans le Tractatus de herbis, l'agnus castus est décrit comme un «arbrisseau semblable à un arbre $»^{23}$, tandis que Pierre de Crescens, dans son traité agronomique, le définit comme un « arbrisseau, c'est-à-dire un tout petit arbre $»^{24}$ - employant alors le terme «arbuscula », peu fréquent dans notre corpus. Ces deux définitions ont en commun d'insister, du fait de critères morphologiques, sur la parenté de cette plante avec les arbres, auxquels elle ne se rattache cependant pas tout à fait. Albert le Grand réaffirme, quant à lui, l'appartenance de ce végétal au monde des arbres en déclarant :

L'agnus castus est un arbre, qui du fait de sa haute taille et de la dureté de ses branches ne peut être décrit autrement que comme un arbre $^{25}$.

Par ailleurs, les traductions vernaculaires des textes botaniques ne font que renforcer cette incertitude de la nomenclature. Le Livre des simples médecines, et l'Arbolayre à sa suite, qui appartiennent à la traduction textuelle du Tractatus de herbis, emploient le terme d'arbricel pour qualifier le gattilier ${ }^{26}$. La traduction française du traité de Pierre de Crescens affirme qu'il s'agit d'un «petit arbret ${ }^{27} »$. L'Hortus sanitatis, enfin, souligne qu'il existe deux espèces d'agnus castus, dont l'une est

\footnotetext{
${ }^{21}$ Vincent de Beauvais, Speculum naturale, 1. XII, c. 47.

22 Thomas de Cantimpré, Liber de natura rerum, 1. X, 5, p. 316.

23 «Frutex similis arbor » Tractatus de herbis, 6, p. 212.

24 «Frutex, id est arbuscula parva», Pierre de Crescens, Ruralia Commoda, 1. V, II, p. 175.

25 «Agnus castus arbor est, quia propter magnitudinem corporis sui et duritiam ramorum non nisi arbor dici potest » Albert le Grand, De vegetabilibus, 1. VI, tract. I, 5 , p. 349.

${ }^{26}$ L'Arbolayre, ou Grant herbier en francoys, est l'un des premiers herbiers imprimés, qui connaît une dizaine d'éditions de 1488 à 1548. Son texte s'inspire en grande partie du Livre des simples médecines.

${ }_{27}^{27}$ Pierre de Crescens, Le Livre des ruraulx prouffitz du labour des champs, 1. V, II, 4.
} 
décrite comme une «plante qui est de la grandeur des arbres ${ }^{28} »$. Cette plante semble pouvoir se rattacher à toutes les classes du règne végétal.

Les incertitudes de la nomenclature sont bien sûr dues à une indétermination présente chez les auctoritates antiques. Dioscoride en parle comme d'un «frutex in arborem assurgens ${ }^{29} »$, un arbrisseau qui se dresse à la manière des arbres, tandis que Pline évoque à son sujet deux espèces, dont l'une est grande et s'élève comme le saule à la hauteur des arbres, tandis que l'autre est plus petite et donc davantage assimilée à l'herbe $^{30}$. Isidore de Séville, quant à lui, passe sous silence le cas de l'agnus castus, ce qui ne facilite pas la tâche aux encyclopédistes médiévaux. Barthélemy cite Pline, mais ne retient que l'une des deux espèces évoquées dans l'Historia naturalis. Vincent de Beauvais, au contraire, s'appuie davantage sur le texte de Dioscoride pour classer cette plante au rang des frutices. Le choix de la source à compiler s'avère donc bien déterminant.

Parmi les végétaux dont l'identité pose problème, nous pouvons également nous arrêter sur le cas de l'herba savina, genévrier sabine en français moderne (Juniperus sabina L selon la nomenclature de Linné.). Son nom latin semble la rattacher au monde des herbes, sans laisser place au doute: elle fait cependant bien partie des végétaux dont la classification n'est guère aisée. Pierre de Crescens la décrit en effet comme un petit arbre, «arbor satis parva ${ }^{31} »$. C'est également le cas dans le Tractatus de herbis ${ }^{32}$. Vincent de Beauvais, au contraire, la classe

\footnotetext{
${ }^{28}$ Ortus sanitatis translaté de latin en français, Paris, pour Antoine Vérard, 1499, 11.

${ }^{29}$ Dioscorides, De materia medica, Colle di Val d'Elsa, Johannes de Medemblick, 1478.

30 «Duo genera eius: maior in arborem salicis modo adsurgit, minor ramosa, foliis candidioribus, lanuginosis. » Pline l'Ancien, Historia naturalis, 1. XXIV, 38, p. 46.

${ }^{31}$ Pierre de Crescens, Ruralia commoda, 1. V, c. 2.

${ }^{32}$ Tractatus de herbis, c. 441, p. 732.
} 
dans le livre IX du Speculum naturale, qui traite des herbes communes ${ }^{33}$. Aucune référence à sa proximité avec les arbres n'y est faite. Si l'on se réfère aux sources antiques, on relève que cette plante porte bien chez Pline le nom d'herba sabina, mais qu'elle figure au livre XXIV de l'Historia naturalis, qui traite des propriétés médicales des arbres. Pline ne revient pas particulièrement sur les questions de nomenclature, mais se concentre sur les vertus thérapeutiques de la plante. Il compare néanmoins ses feuilles à celles d'autres végétaux, tels que le tamaris ou le cyprès de Crète: il s'agit donc d'une herbe qui présente des ressemblances physiques avec les arbres ${ }^{34}$. Une fois encore, les critères morphologiques rencontrent leur limite quand il s'agit d'ordonner le monde végétal.

L'incertitude quant à la catégorie à laquelle appartient ce végétal peut même se refléter dans les marques d'usage et les pratiques de lecture. Certains manuscrits du Livre des simples médecines portent pour ce chapitre un titre rubriqué «De ung arbre ou herbe dit savignier $»^{35}$, mettant par là l'accent sur son ambivalence. De même, un exemplaire imprimé du De viribus herbarum de Macer Floridus comporte, au chapitre consacré à l'herba savina, la note marginale suivante: «est arbor, non herba ${ }^{36} »$, tandis que la mention herba dans le texte a été barrée. Une étude plus approfondie des notes marginales et autres marques d'usage de ces ouvrages pourrait permettre de mieux appréhender la question de la nomenclature botanique : elles sont en effet révélatrices de la réception de ces classifications par le lectorat des

\footnotetext{
${ }^{33}$ Vincent de Beauvais, Speculum naturale, 1. IX, c. 128.

${ }^{34}$ Pline 1'Ancien, Historia naturalis, 1. XXIV, 61, p. 60.

${ }^{35}$ Paris, BnF, fr. 9136, f. 265v.

${ }^{36}$ Macer Floridus, De viribus herbarum famosissimus medicus et medicorum speculum, [Genève, Louis Cruse, vers 1500]. Paris, BnF, Rés. $4^{\circ}$ Te142 (4), f. 12. Précisons par ailleurs que cette compilation poétique datant du dernier quart $\mathrm{du}$ $\mathrm{XI}^{\mathrm{e}}$ siècle ne traite que de plantes herbacées et n'évoque pas les arbres.
} 
herbiers, qui, tout comme les auteurs des textes, peut poser un regard critiques sur les sources du savoir naturel.

Ordonner le monde des plantes: telle est donc l'ambition des encyclopédies, ces «cathédrales magnifiquement architecturées ${ }^{37}$ ». Les herbiers médiévaux sont bien révélateurs tant de l'appréhension raisonnée que les naturalistes médiévaux ont du monde végétal que de leur rapport aux textes qu'ils compilent. Les plantes à l'identité ambivalente, à michemin entre le monde des herbes et celui des arbres, permettent de révéler ces mécanismes propres à la littérature botanique médiévale. Les encyclopédistes ne se limitent pas au seul rôle de compilateur, mais font des choix parmi les sources qu'ils traitent et redessinent par là le paysage de l'ordonnancement du monde végétal.

Toute tentative de déterminer des critères morphologiques semble donc contrainte face à la foisonnante diversité de la flore. Ces limites ne sont cependant pas propres à la philosophie naturelle médiévale, mais irriguent toute l'histoire de la botanique. « Ce n'est pas une tâche aisée que de définir l'arbre ${ }^{38} »$, écrit le botaniste Francis Hallé dans son Plaidoyer pour l'arbre, réaffirmant ainsi le caractère mouvant de cette catégorie reine du monde végétal, si familière et pourtant si difficile à saisir et à classer.

\footnotetext{
${ }^{37}$ Éducation et cultures : Occident chrétien, XII $-m i-X V^{e}$ siècle, éd. Isabelle HeullantDonat, Neuilly-sur-Seine, Atlande, 1999, p. 554.

${ }^{38}$ Francis Hallé, Plaidoyer pour l'arbre, Arles, Actes Sud, 2005, p. 17.
} 